\title{
The difference in age of the two counter-rotating stellar disks of the spiral galaxy NGC $4138^{\star}$
}

\author{
A. Pizzella ${ }^{1,2}$, L. Morelli ${ }^{1,2}$, E. M. Corsini ${ }^{1,2}$, E. Dalla Bontà ${ }^{1,2}$, L. Coccato $^{3}$, and G. Sanjana ${ }^{4}$ \\ 1 Dipartimento di Fisica e Astronomia "G. Galilei”, Università di Padova, vicolo dell’Osservatorio 3, 35122 Padova, Italy \\ e-mail: alessandro.pizzella@unipd.it \\ 2 INAF-Osservatorio Astronomico di Padova, vicolo dell'Osservatorio 5, 35122 Padova, Italy \\ 3 European Southern Observatory, Karl-Schwarzschild-Straße 2, 85748 Garching bei München, Germany \\ ${ }^{4}$ Department of Physics, Imperial College London, South Kensington Campus, London SW7 2AZ, UK
}

Received 4 August 2014 / Accepted 5 September 2014

\section{ABSTRACT}

\begin{abstract}
Context. Galaxies accrete material from the environment through acquisitions and mergers. These processes contribute to the galaxy assembly and leave their fingerprints on the galactic morphology, internal kinematics of gas and stars, and stellar populations. Aims. The Sa spiral NGC 4138 is known to host two counter-rotating stellar disks, with the ionized gas co-rotating with one of them. We measured the kinematics and properties of the two counter-rotating stellar populations to constrain their formation scenario. Methods. A spectroscopic decomposition of the observed major-axis spectrum was performed to disentangle the relative contribution of the two counter-rotating stellar and one ionized-gas components. The line-strength indices of the two counter-rotating stellar components were measured and modeled with single stellar population models that account for the $\alpha / \mathrm{Fe}$ overabundance.

Results. The counter-rotating stellar population is younger, marginally more metal poor, and more $\alpha$-enhanced than the main stellar component. The younger stellar component is also associated with a star-forming ring.

Conclusions. The different properties of the counter-rotating stellar components of NGC 4138 rule out the idea that they formed because of bar dissolution. Our findings support the results of numerical simulations in which the counter-rotating component assembled from gas accreted on retrograde orbits from the environment or from the retrograde merging with a gas-rich dwarf galaxy.
\end{abstract}

Key words. galaxies: abundances - galaxies: spiral - galaxies: kinematics and dynamics - galaxies: formation galaxies: stellar content - galaxies: individual: NGC 4138

\section{Introduction}

Counter-rotating galaxies host two components rotating in opposite directions with respect to each other (Bertola \& Corsini 1999). Large-scale counter-rotating disks of stars and/or gas have been detected in several lenticular and spiral galaxies, and different mechanisms have been proposed to explain their formation (Corsini 2014). They are expected to leave different signatures in the properties of the counter-rotating stellar populations. In particular, their age difference may be used to discriminate between competing scenarios for the origin of counterrotation. Gas accretion followed by star formation always predicts a younger age for the counter-rotating component (Pizzella et al. 2004; Vergani et al. 2007; Algorry et al. 2014), whereas the counter-rotating component formed by the retrograde capture of stars through minor or major mergers may be either younger or older with respect to the pre-existing stellar disk (Thakar et al. 1997; Crocker et al. 2009). The external origin also allows the two counter-rotating components to have different metallicities and $\alpha$-enhancements. In contrast, the formation of large-scale counter-rotating stellar disks due to bar dissolution predicts the same mass, chemical composition, and age for both the prograde and retrograde components (Evans \& Collett 1994).

We developed a spectroscopic decomposition that separates the relative contribution of the counter-rotating stellar components to the observed galaxy spectrum in order to constrain the

^ Based on observation carried out at the Galileo $1.22 \mathrm{~m}$ telescope at Padua University. properties of the counter-rotating stellar populations (Coccato et al. 2011). We successfully applied it to the counter-rotating stellar disks of NGC 3593, NGC 4550, and NGC 5719 (Coccato et al. 2011, 2013). In all of them, the counter-rotating stellar disk rotates in the same direction as the ionized gas, and it is less massive, younger, more metal poor, and more $\alpha$-enhanced than the main stellar disk. These findings rule out an internal origin of the secondary stellar component and favor a scenario where it formed from gas accreted on retrograde orbits from the environment, which fueled rapid star formation. The merging scenario cannot be ruled out, because it also allows the counter-rotating component to be the younger one in half of the cases. The available statistics are not sufficient to draw significant conclusions and therefore a larger sample is required to address this issue.

In this paper our spectroscopic technique is applied to derive the properties of the counter-rotating stellar populations of the Sa galaxy NGC 4138. The structure, kinematics, and dynamics of this nearby and relatively isolated galaxy were studied in detail by Jore et al. (1996) and Afanasiev \& Sil'chenko (2002). Optical images show no major peculiarities except for a narrow dust lane in the galactic disk. Radio observations show an extended and warped disk of neutral hydrogen which rotates in the same direction as the ionized gas and counter-rotates with respect to the main stellar component (Jore et al. 1996).

This paper is organized as follows: the spectroscopic data of NGC 4138 are presented in Sect. 2. The kinematic and stellar population properties of the galaxy are measured and discussed in Sect. 3. The conclusions about the formation scenario of the counter-rotating components of NGC 4138 are given in Sect. 4. 


\section{Observations and data reduction}

The spectroscopic observations of NGC 4138 were carried out at the Asiago Astrophysical Observatory of Padua University with the $1.22 \mathrm{~m}$ Galileo telescope on December 12, 2012. The telescope was equipped with the Cassegrain Boller \& Chivens spectrograph. The grating with 1200 grooves $\mathrm{mm}^{-1}$ was used in the first order in combination with a 3 .' $^{\prime} \times 7.75$ slit and an Andor iDus DU440 CCD, which has $2048 \times 512$ pixels of $26 \times 26 \mu \mathrm{m}^{2}$. The gain and readout noise were $0.97 \mathrm{e}^{-}$count $^{-1}$ and $3.4 \mathrm{e}^{-}$(rms), respectively. The spectral range between about $4500 \AA$ and $5700 \AA$ was covered with a reciprocal dispersion of $0.60 \AA$ pixel $^{-1}$. The spatial scale was $1^{\prime \prime}$. 0 pixel $^{-1}$. The instrumental resolution was $2.47 \pm 0.18 \AA$ (FWHM), and it was derived from the mean of the Gaussian FWHM that were measured for a dozen unblended sky emission lines distributed over the whole spectral range of a wavelength-calibrated spectrum. It corresponds to $\sigma_{\text {instr }}=63 \pm 4 \mathrm{~km} \mathrm{~s}^{-1}$ at $5000 \AA$.

The galaxy was observed along the major axis $\left(\mathrm{PA}=150^{\circ}\right)$. At the beginning of each exposure, the galaxy was centered on the slit using the guiding camera. Seven exposures of $1800 \mathrm{~s}$ each ensured $3.5 \mathrm{~h}$ of effective integration. HR8634 was observed as spectro-photometric standard star to calibrate the flux of the galaxy spectra before line-strength indices were measured. Spectra of the comparison arc lamp were taken before and after object exposures. The value of the seeing FWHM during the observing nights, as measured on the guiding star, was about $4 "$.

All the spectra were bias subtracted, flat-field corrected, cleaned of cosmic rays, corrected for bad columns, and wavelength and flux calibrated using $\mathrm{IRAF}^{1}$. Each spectrum was rebinned using the wavelength solution obtained from the corresponding arc-lamp spectrum obtained as the average of the arclamp observed immediately before and after the science exposure. All the galaxy and stellar spectra were corrected for CCD misalignment. The sky contribution was determined by interpolating along the two edges of the slit, where the stellar light of the galaxy was negligible, and then subtracted. The different galaxy spectra were co-added into a single major-axis spectrum using the center of the stellar continuum as a reference.

\section{Analysis and results}

The galaxy spectrum was first analyzed without disentangling the two counter-rotating components; afterwards the stellar population properties of both components were derived by performing a spectroscopic decomposition.

\subsection{Single-component analysis and results}

The stellar kinematics (Fig. 1) were measured following Pizzella et al. (2013) using the Penalized Pixel Fitting (pPXF, Cappellari \& Emsellem 2004) and Gas and Absorption Line Fitting (GANDALF; Sarzi et al. 2006) IDL ${ }^{2}$ codes. The galaxy spectrum was rebinned along the dispersion direction to a logarithmic scale and along the spatial direction to obtain a signal-tonoise ratio $S / N \geq 15$ per resolution element when measuring the kinematic parameter. At each radius, a linear combination

\footnotetext{
1 Image Reduction and Analysis Facility (IRAF) is distributed by the National Optical Astronomy Observatories, which are operated by the Association of Universities for Research in Astronomy, Inc., under cooperative agreement with the National Science Foundation.

2 The Interactive Data Language (IDL) is distributed by ITT Visual Information Solutions.
}

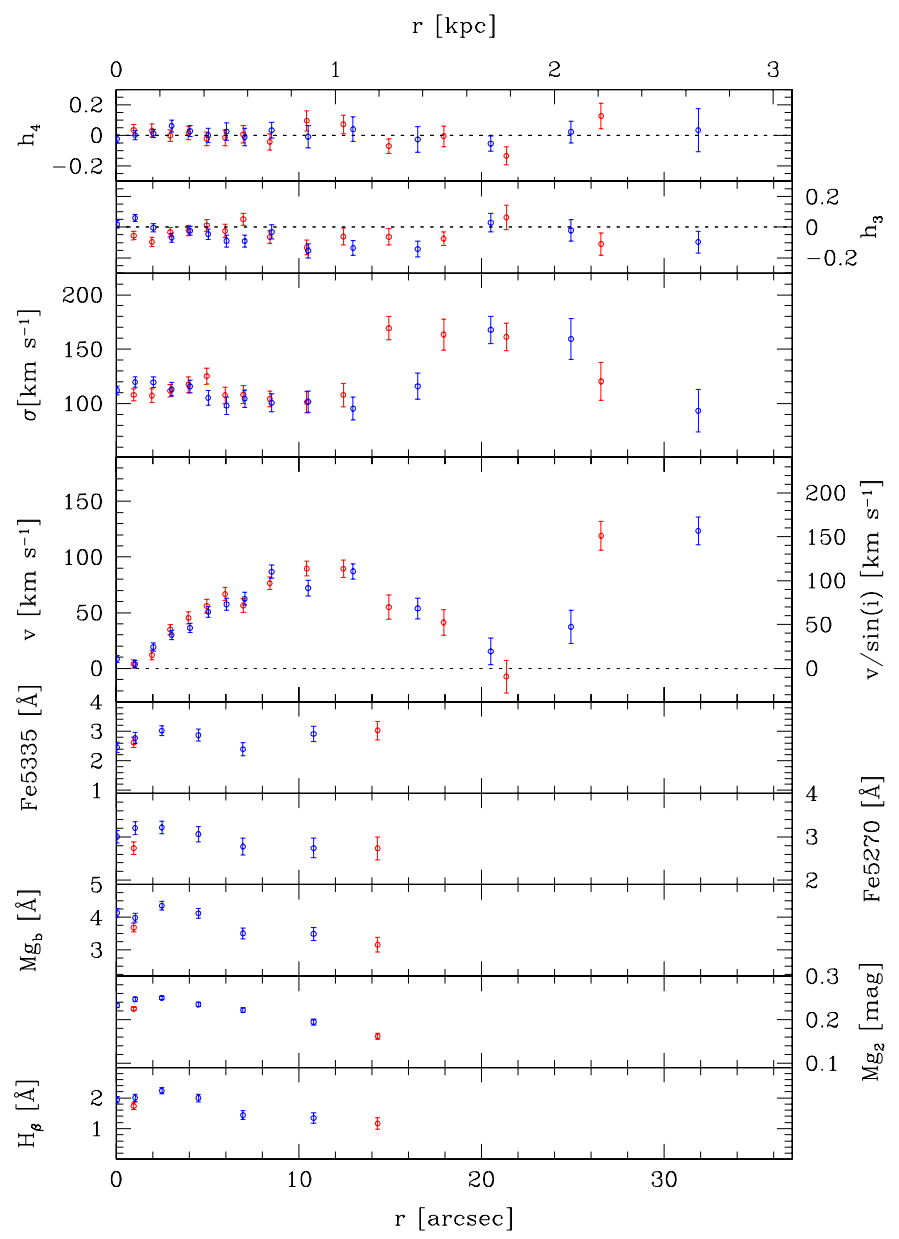

Fig. 1. Stellar kinematics and line-strength indices as measured along the major axis of NGC 4138. The radial profiles of the line-of-sight fourth- and third-order coefficient of the Gauss-Hermite decomposition of the LOSVD $\left(h_{4}\right.$ and $\left.h_{3}\right)$; velocity dispersion $(\sigma)$; velocity $(v)$ after the subtraction of systemic velocity; and the line-strength indices $\mathrm{Fe}_{5335}$, $\mathrm{Fe}_{5270}, \mathrm{Mg} b, \mathrm{Mg}_{2}$, and $\mathrm{H} \beta$ are plotted (from top to bottom). The red and blue dots indicate the receding (NW) and approaching (SE) side of the galaxy, respectively.

of single stellar population models from Maraston \& Strömbäck (2011) was convolved with the line-of-sight velocity distribution (LOSVD) assumed to be a Gaussian plus third- and fourth-order Gauss-Hermite polynomials and fitted to the galaxy spectrum by $\chi^{2}$ minimization in pixel space.

The stellar kinematics are given in Table 1 and extend out to $|r| \simeq 30^{\prime \prime}$ on both sides of the nucleus. The velocity curve is symmetric around the center and the deprojected velocity rises to $\simeq 100 \mathrm{~km} \mathrm{~s}^{-1}$ at $|r| \simeq 12^{\prime \prime}$. Farther out it decreases to $\sim 0 \mathrm{~km} \mathrm{~s}^{-1}$ at $|r| \simeq 21^{\prime \prime}$. For larger radii the velocity increases again to $\simeq 100 \mathrm{~km} \mathrm{~s}^{-1}$. The velocity dispersion is $\simeq 120 \mathrm{~km} \mathrm{~s}^{-1}$ in the center and slightly decreases outwards. It increases again for $|r|>14^{\prime \prime}$ peaking at $\simeq 170 \mathrm{~km} \mathrm{~s}^{-1}$ at $|r| \simeq 21^{\prime \prime}$. This behavior of both the velocity and velocity dispersion radial profiles is the signature of the presence of a counter-rotating stellar component (Bertola et al. 1996; Vergani et al. 2007). The relative contribution of the counter-rotating component to the galaxy surface brightness is maximum at $|r| \simeq 21^{\prime \prime}$ (Jore et al. 1996). In this radial range a star-forming ring of gas (Pogge \& Eskridge 1987; Jore et al. 1996) and stars (Jore et al. 1996; Afanasiev $\&$ Sil'chenko 2002) was detected. The main stellar component dominates the light distribution of the inner regions $\left(|r|<12^{\prime \prime}\right)$. 
Table 1. Stellar kinematics along the major axis of NGC 4138.

\begin{tabular}{rrrrr}
\hline \hline \multicolumn{1}{c}{$r$} & \multicolumn{1}{c}{$V$} & \multicolumn{1}{c}{$\sigma$} & \multicolumn{1}{c}{$h_{3}$} & \multicolumn{1}{c}{$h_{4}$} \\
{$\left[{ }^{\prime \prime}\right]$} & {$\left[\mathrm{km} \mathrm{s}^{-1}\right]$} & {$\left[\mathrm{km} \mathrm{s}^{-1}\right]$} & & \\
\hline-31.9 & $-124 \pm 12$ & $93 \pm 20$ & $0.097 \pm 0.071$ & $0.034 \pm 0.141$ \\
-24.9 & $-37 \pm 15$ & $159 \pm 19$ & $0.020 \pm 0.070$ & $0.022 \pm 0.072$ \\
-20.5 & $-15 \pm 12$ & $168 \pm 13$ & $-0.030 \pm 0.061$ & $-0.054 \pm 0.050$ \\
-16.5 & $-54 \pm 9$ & $116 \pm 12$ & $0.142 \pm 0.051$ & $-0.026 \pm 0.084$ \\
-13.0 & $-87 \pm 7$ & $95 \pm 10$ & $0.136 \pm 0.048$ & $0.040 \pm 0.080$ \\
-10.5 & $-72 \pm 7$ & $102 \pm 10$ & $0.154 \pm 0.046$ & $-0.009 \pm 0.073$ \\
-8.5 & $-87 \pm 6$ & $101 \pm 8$ & $0.031 \pm 0.047$ & $0.033 \pm 0.052$ \\
-7.0 & $-63 \pm 6$ & $104 \pm 8$ & $0.091 \pm 0.040$ & $-0.012 \pm 0.057$ \\
-6.0 & $-58 \pm 5$ & $98 \pm 8$ & $0.090 \pm 0.039$ & $0.025 \pm 0.058$ \\
-5.0 & $-51 \pm 5$ & $105 \pm 7$ & $0.045 \pm 0.034$ & $0.001 \pm 0.047$ \\
-4.0 & $-36 \pm 4$ & $116 \pm 6$ & $0.023 \pm 0.030$ & $0.028 \pm 0.035$ \\
-3.0 & $-30 \pm 4$ & $113 \pm 6$ & $0.068 \pm 0.029$ & $0.062 \pm 0.039$ \\
-2.0 & $-19 \pm 4$ & $120 \pm 5$ & $0.004 \pm 0.026$ & $0.013 \pm 0.028$ \\
-1.0 & $-4 \pm 3$ & $120 \pm 5$ & $-0.061 \pm 0.024$ & $0.002 \pm 0.030$ \\
-0.0 & $-8 \pm 3$ & $112 \pm 4$ & $-0.020 \pm 0.025$ & $-0.023 \pm 0.026$ \\
1.0 & $4 \pm 4$ & $108 \pm 6$ & $-0.055 \pm 0.027$ & $0.035 \pm 0.036$ \\
2.0 & $12 \pm 4$ & $107 \pm 6$ & $-0.097 \pm 0.031$ & $0.031 \pm 0.043$ \\
3.0 & $35 \pm 4$ & $112 \pm 6$ & $-0.032 \pm 0.031$ & $-0.001 \pm 0.037$ \\
4.0 & $45 \pm 6$ & $118 \pm 7$ & $-0.022 \pm 0.034$ & $0.016 \pm 0.046$ \\
5.0 & $56 \pm 6$ & $125 \pm 8$ & $0.012 \pm 0.038$ & $-0.022 \pm 0.045$ \\
6.0 & $67 \pm 6$ & $108 \pm 8$ & $-0.024 \pm 0.042$ & $-0.015 \pm 0.052$ \\
7.0 & $56 \pm 6$ & $108 \pm 8$ & $0.052 \pm 0.039$ & $0.007 \pm 0.057$ \\
8.4 & $76 \pm 6$ & $104 \pm 7$ & $-0.064 \pm 0.041$ & $-0.042 \pm 0.053$ \\
10.4 & $90 \pm 7$ & $101 \pm 10$ & $-0.133 \pm 0.048$ & $0.096 \pm 0.066$ \\
12.4 & $90 \pm 8$ & $108 \pm 11$ & $-0.062 \pm 0.055$ & $0.072 \pm 0.061$ \\
14.9 & $55 \pm 11$ & $169 \pm 11$ & $-0.063 \pm 0.054$ & $-0.070 \pm 0.047$ \\
17.9 & $41 \pm 12$ & $164 \pm 14$ & $-0.074 \pm 0.045$ & $-0.006 \pm 0.067$ \\
21.4 & $-7 \pm 15$ & $161 \pm 13$ & $0.064 \pm 0.080$ & $-0.133 \pm 0.058$ \\
26.6 & $119 \pm 13$ & $120 \pm 17$ & $-0.109 \pm 0.073$ & $0.127 \pm 0.084$ \\
\hline & & & &
\end{tabular}

The $\mathrm{Mg}, \mathrm{Fe}$, and $\mathrm{H} \beta$ line-strength indices (Fig. 1) were measured from the absorption features of the galaxy spectrum following Morelli et al. (2012). The galaxy spectrum was rebinned in the radial direction to achieve a $S / N \geq 25$ per resolution element. The spectral resolution of the galaxy spectrum was degraded through a Gaussian convolution to match the Lick/IDS resolution before measuring the line-strength indices.

The values of the line-strength indices measured for a sample of templates were compared to those obtained by Worthey (1994) to calibrate our measurements to the Lick/IDS system. The offsets were neglected, being smaller than the mean error of the differences. The average iron index $\langle\mathrm{Fe}\rangle$ (Gorgas et al. 1990) and combined magnesium-iron index $[\mathrm{MgFe}]^{\prime}$ (Thomas et al. 2003) were calculated to be used in combination with the $\operatorname{Mg} b$ and $\mathrm{H} \beta$ indices to constrain the stellar population properties.

A foreground star is observed at $r \simeq 4^{\prime \prime}$ NW from the center of NGC 4138 roughly along its major axis (Afanasiev \& Sil'chenko 2002). The star spectrum affects the line-strength measurements since it changes the observed continuum level, lowering the strength of the galaxy absorption features. The large difference between the redshifts of the star and galaxy allowed the correct measurement of the galaxy kinematics. Only the linestrength measurements not affected by the foreground star are shown in Fig. 1. They are listed in Table 2 and are found to be in agreement with the measurements by Afanasiev \& Sil'chenko (2002).

The central line-strength indices fit within the scatter the $\mathrm{Mg}_{2}-\sigma,\langle\mathrm{Fe}\rangle-\sigma$, and $\mathrm{H} \beta-\sigma$ relations for early-type spiral galaxies by Morelli et al. (2012).
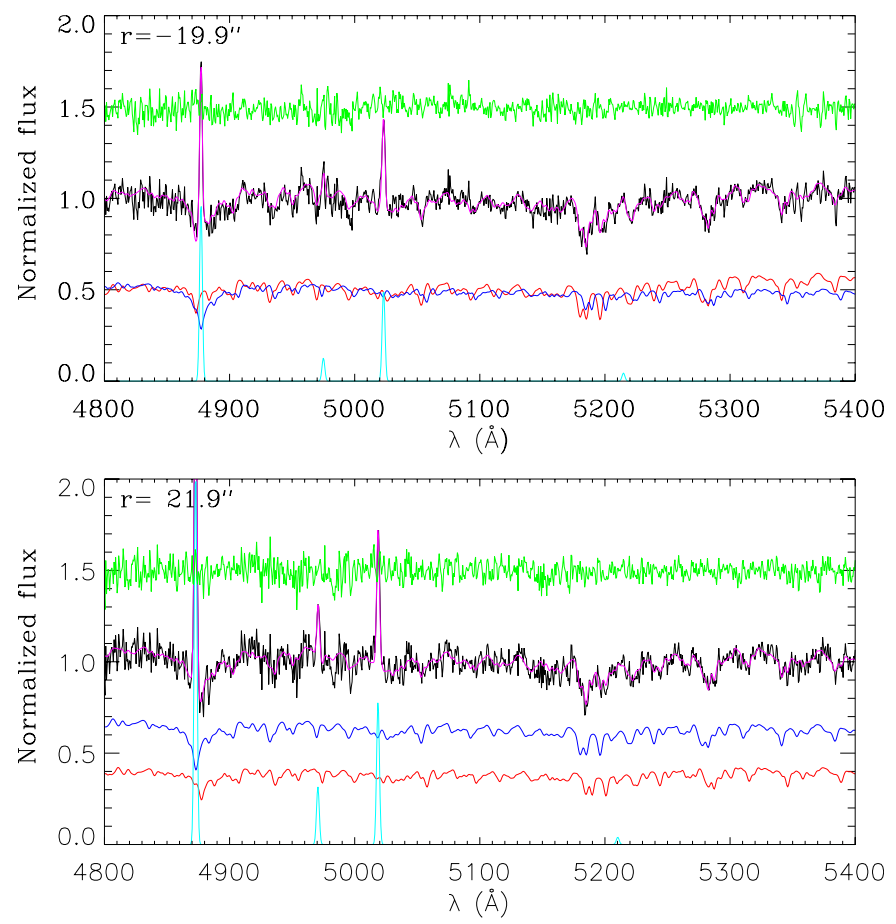

Fig. 2. Fit of the averaged spectrum (black line) in the spatial bin at $-19.9^{\prime \prime}$ (top panel) and +21.9" (bottom panel) from the galaxy center. The best-fitting model (magenta line) is the sum of the spectra of the ionized-gas component (cyan line) and the two stellar components (blue and red lines). The normalized flux of the fit residual (green line) has a false zero point for viewing convenience. The double peaked absorption lines are evident in the averaged spectra.

Table 2. Line-strength indices along the major axis of NGC 4138.

\begin{tabular}{rccccc}
\hline \hline \multicolumn{1}{c}{$r$} & $\mathrm{H} \beta$ & $\mathrm{Mg}_{2}$ & $\mathrm{Mg} b$ & $\mathrm{Fe}_{5270}$ & $\mathrm{Fe}_{5335}$ \\
{$\left[{ }^{\prime \prime}\right]$} & {$[\AA]$} & {$[\mathrm{mag}]$} & {$[\AA]$} & {$[\AA]$} & {$[\AA]$} \\
\hline-10.8 & $1.35 \pm 0.17$ & $0.20 \pm 0.01$ & $3.49 \pm 0.20$ & $2.74 \pm 0.23$ & $2.91 \pm 0.27$ \\
-6.9 & $1.44 \pm 0.14$ & $0.22 \pm 0.01$ & $3.51 \pm 0.17$ & $2.77 \pm 0.19$ & $2.39 \pm 0.23$ \\
-4.5 & $2.00 \pm 0.13$ & $0.24 \pm 0.01$ & $4.12 \pm 0.15$ & $3.06 \pm 0.17$ & $2.87 \pm 0.21$ \\
-2.5 & $2.24 \pm 0.10$ & $0.25 \pm 0.01$ & $4.36 \pm 0.13$ & $3.21 \pm 0.14$ & $3.02 \pm 0.17$ \\
-1.0 & $2.01 \pm 0.12$ & $0.25 \pm 0.01$ & $3.99 \pm 0.14$ & $3.20 \pm 0.15$ & $2.77 \pm 0.19$ \\
-0.0 & $1.94 \pm 0.11$ & $0.23 \pm 0.01$ & $4.14 \pm 0.13$ & $3.00 \pm 0.14$ & $2.45 \pm 0.18$ \\
1.0 & $1.73 \pm 0.11$ & $0.23 \pm 0.01$ & $3.69 \pm 0.13$ & $2.74 \pm 0.14$ & $2.62 \pm 0.18$ \\
14.3 & $1.17 \pm 0.18$ & $0.16 \pm 0.01$ & $3.16 \pm 0.22$ & $2.73 \pm 0.26$ & $3.03 \pm 0.31$ \\
\hline
\end{tabular}

\subsection{Two-component analysis and results}

A high spectral $\mathrm{S} / \mathrm{N}$ is needed to perform a spectral decomposition. Therefore, we averaged the galaxy spectrum along the spatial direction between $r=-25^{\prime \prime}$ and $r=-16^{\prime \prime}$, and between $r=17^{\prime \prime}$ and $r=28^{\prime \prime}$ (Fig. 2). The resulting spectra sample the two radial ranges where the contribution of the counter-rotating component is at its maximum.

The two spectra were analyzed with the novel implementation pPXF developed in Coccato et al. (2011). It fitted to the galaxy spectrum two synthetic templates (one for each stellar component) obtained as a linear combination of models by Maraston \& Strömbäck (2011) by convolving them with two Gaussian LOSVDs with different kinematics. The relative contribution of the counter-rotating components to the total spectrum was in terms of light. Gaussian functions were added to the convolved synthetic templates to account for ionized-gas 

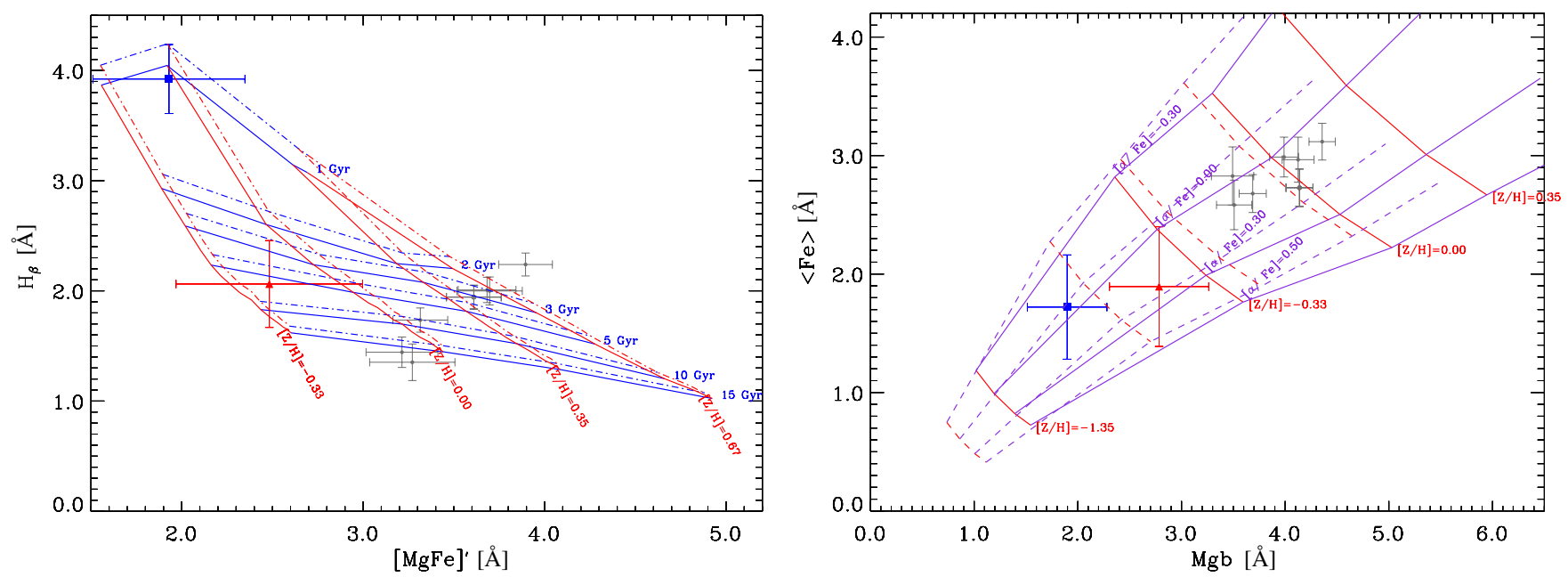

Fig. 3. $\mathrm{H} \beta$ and $[\mathrm{MgFe}]^{\prime}$ indices (left panel) and $\mathrm{Mg} b$ and $\langle\mathrm{Fe}\rangle$ indices (right panel) measured along the major axis of NGC 4138 . The red triangles and blue squares correspond to the values measured at $|r| \simeq 21^{\prime \prime}$ for the co-rotating and counter-rotating components, respectively. The gray filled circles are the line strengths measured in the inner $12^{\prime \prime}$ where only the main component is present. The center of the galaxy is indicated by an open gray circle. The lines indicate the models by Thomas et al. (2003). In the left panel the age-metallicity grids are plotted with two different $\alpha /$ Fe enhancements: $[\alpha / \mathrm{Fe}]=0.0 \mathrm{dex}$ (continuous lines) and $[\alpha / \mathrm{Fe}]=0.5 \mathrm{dex}$ (dashed lines). In the right panel the $[\alpha / \mathrm{Fe}]$ ratio-metallicity grids are plotted with two different ages: 2 Gyr (continuous lines) and 12 Gyr (dashed lines).

Table 3. Line-strength indices at $|r| \simeq 21^{\prime \prime}$ for the co-rotating (No. 1) and counter-rotating (No. 2) stellar components of NGC 4138.

\begin{tabular}{cccccc}
\hline \hline No. & $\begin{array}{c}\mathrm{H} \beta \\
{[\AA]}\end{array}$ & $\begin{array}{c}\mathrm{Mg}_{2} \\
{[\mathrm{mag}]}\end{array}$ & $\begin{array}{c}\mathrm{Mg} b \\
{[\AA]}\end{array}$ & $\begin{array}{c}\mathrm{Fe}_{5270} \\
{[\AA]}\end{array}$ & $\begin{array}{c}\mathrm{Fe}_{5335} \\
{[\AA]}\end{array}$ \\
\hline 1 & $2.06 \pm 0.39$ & $0.18 \pm 0.01$ & $2.78 \pm 0.48$ & $2.14 \pm 0.47$ & $1.65 \pm 0.54$ \\
2 & $3.92 \pm 0.31$ & $0.10 \pm 0.01$ & $1.90 \pm 0.38$ & $1.85 \pm 0.39$ & $1.59 \pm 0.49$ \\
\hline
\end{tabular}

emission lines. The spectroscopic decomposition returned the spectra of two best-fit synthetic stellar templates and ionizedgas emissions, along with the best-fitting parameters of luminosity fraction and line-of-sight velocity and velocity dispersion. In both spatial bins the averaged spectrum is the superposition of two stellar components which are rotating in opposite directions (Fig. 2). One component is co-rotating with the ionized gas and main body of the galaxy, while the other one is counter-rotating. In particular we measured a mean velocity of $-116 \pm 5 \mathrm{~km} \mathrm{~s}^{-1}$ at $r=-19^{\prime} .9$ and $153 \pm 8 \mathrm{~km} \mathrm{~s}^{-1}$ at $r=+21^{\prime \prime} .9$ for the main component and a mean velocity of $135 \pm 10 \mathrm{~km} \mathrm{~s}^{-1}$ at $r=-19.9$ and $-117 \pm 8 \mathrm{~km} \mathrm{~s}^{-1}$ at $r=+21^{\prime \prime} .9$ for the secondary component. The ionized gas has about the same velocity as the counter-rotating component (Fig. 2).

Both components contribute about $50 \%$ of the galaxy luminosity in the binned radial range. The line-of-sight velocities and velocity dispersions measured for the ionized gas and the two stellar components are consistent with Jore et al. (1996).

The line-strength indices of the two counter-rotating components were extracted from the two best-fit synthetic templates. The values measured for each component on the two galaxy sides were averaged and are given in Table 3. In Fig. 3 these measurements are compared to the line-strength indices predicted for a single stellar population as a function of the age, metallicity, and $[\alpha / \mathrm{Fe}]$ ratio by Thomas et al. (2003). The line-strength indices derived in the inner $12^{\prime \prime}$ where the main stellar component is dominant are also plotted in Fig. 3. They are representative of the main body of the galaxy.
The two counter-rotating stellar components are characterized by marginally different chemical properties. The co-rotating stellar component is characterized by higher values of $[\mathrm{MgFe}]^{\prime}$, $\mathrm{Mg} b$ and $\langle\mathrm{Fe}\rangle$, and a lower $\mathrm{H} \beta$ value with respect to the counterrotating component. This translates into different properties of their stellar populations, which were derived by a linear interpolation of measured line strengths between the model predictions using an iterative procedure as in Morelli et al. (2008). The corotating stellar component is older $(6.6 \pm 3.6 \mathrm{Gyr})$ with sub-solar metallicity $([\mathrm{Z} / \mathrm{H}]=-0.24 \pm 0.46 \mathrm{dex})$ and super-solar enhancement $([\alpha / \mathrm{Fe}]=0.24 \pm 0.19 \mathrm{dex})$, whereas the counter-rotating stellar population component is younger $(1.1 \pm 0.3 \mathrm{Gyr})$ with solar metallicity $([\mathrm{Z} / \mathrm{H}]=-0.04 \pm 0.27 \mathrm{dex})$ and solar enhancement $([\alpha / \mathrm{Fe}]=0.08 \pm 0.21 \mathrm{dex})$. The age of the co-rotating component matches the age range (2-15 Gyr) measured for the main body of the galaxy.

\section{Discussion and conclusions}

The Sa spiral NGC 4138 hosts two cospatial counter-rotating stellar disks, one of which is co-rotating with the gaseous disk (Jore et al. 1996; Afanasiev \& Sil'chenko 2002).

We have decomposed an intermediate-resolution spectrum measured along the major axis of NGC 4138 to derive the properties of the counter-rotating stellar populations at $|r| \simeq 21^{\prime \prime}$. In this radial range the counter-rotating components give about the same contribution to the surface brightness of the galaxy. We have found that the counter-rotating stars are on average younger, more metal poor, and more $\alpha$-enhanced than those in the main component. These features were also observed in the few other galaxies where the stellar populations of counterrotating stellar disks of comparable sizes were successfully disentangled (NGC 3593, Coccato et al. 2013; NGC 4550, Coccato et al. 2013 and Johnston et al. 2013; NGC 5719, Coccato et al. 2011). The difference in age between the two counter-rotating stellar components is particularly strong in NGC 5179, which shows a spectacular on-going interaction with its nearby companion NGC 5713. The two galaxies are connected by a tidal bridge of neutral hydrogen which is feeding the counter-rotating gaseous and stellar components (Vergani et al. 2007). On the 
contrary, NGC 3593 and NGC 4550 are both quite isolated and undisturbed galaxies. The age difference between the two stellar populations is less pronounced in NGC 4550, whereas NGC 3593 represents an intermediate case.

The counter-rotating stellar populations of NGC 4138 have almost the same age as those detected in NGC 3593. It is worth noticing that the two galaxies are characterized by similar morphological and structural properties. Indeed, they are very early-type spirals with smooth arms defined by dust lanes (Sandage \& Bedke 1994). Moreover, both NGC 4138 (Pogge \& Eskridge 1987; Jore et al. 1996; Afanasiev \& Sil'chenko 2002) and NGC 3593 (Corsini et al. 1998; García-Burillo et al. 2000; Coccato et al. 2013) show a star-forming ring of gas and stars in the region where the younger stellar component is detected.

The suppression of arms in counter-rotating spirals has been recently recognized in high-resolution $N$-body simulations of multi-armed spiral features triggered through swing amplification by density inhomogeneities orbiting the disk (D'Onghia et al. 2013). However, a survey of a sample of early-type spirals selected to have the spiral pattern traced by dust lanes revealed the presence of kinematically decoupled gas components but no new case of counter-rotation (Corsini et al. 2003).

The difference in age of the counter-rotating components of NGC 4138 rules out the possibility that their formation was driven by internal mechanisms and supports a process involving acquisition of material from the environment and merging events. Using numerical simulations, Thakar et al. (1997) modeled the formation process of NGC 4138 by investigating the continuous retrograde infall of gas and the retrograde merger with a gas-rich dwarf galaxy. Both processes are successful in producing a counter-rotating disk of the observed mass and size without heating up the primary disk significantly. The timescale for the case of continuous infall ( $5 \mathrm{Gyr}$ ) was found to be somewhat longer than for a dwarf merger ( $4 \mathrm{Gyr})$, but it is still consistent with the age difference actually measured for the two counter-rotating populations. According to the simulations by Thakar et al. (1997), the ring of NGC 4138 was created by counter-rotating gas clouds colliding with co-rotating gas already present in the disk and forming stars in the process. This scenario is supported by our findings that the star-forming ring is associated with the younger stellar component.

A few decades after they were discovered, counter-rotating galaxies still represent a challenging subject. Deriving the properties of their stellar populations with spectral decomposition makes it possible to test the predictions of the different scenarios suggested to explain their formation.

Acknowledgements. This work was supported by Padua University through grants 60A02-5052/11, 60A02-4807/12, 60A02-5857/13, and CPDA133894. L.M. received financial support from Padua University grant CPS0204. G.S. acknowledges Padua University for their hospitality while this paper was in progress.

\section{References}

Afanasiev, V. L., \& Sil'chenko, O. K. 2002, AJ, 124, 706

Algorry, D. G., Navarro, J. F., Abadi, M. G., et al. 2014, MNRAS, 437, 3596

Bertola, F., \& Corsini, E. M. 1999, in Galaxy Interactions at Low and High Redshift, IAU Symp. 186, eds. J. E. Barnes, \& D. B. Sanders (Dordrecht: Kluwer), 149

Bertola, F., Cinzano, P., Corsini, E. M., et al. 1996, ApJ, 458, L67

Cappellari, M., \& Emsellem, E. 2004, PASP, 116, 138

Coccato, L., Morelli, L., Corsini, E. M., et al. 2011, MNRAS, 412, L113

Coccato, L., Morelli, L., Pizzella, A., et al. 2013, A\&A, 549, A3

Corsini, E. M. 2014, in Multi-Spin Galaxies eds. E. Iodice, \& E. M. Corsini (San Francisco: ASP), ASP Conf. Ser., 486, 51

Corsini, E. M., Pizzella, A., Funes, J. G., Vega Beltrán, J. C., \& Bertola, F. 1998, A\&A, 337, 80

Corsini, E. M., Pizzella, A., Coccato, L., \& Bertola, F. 2003, A\&A, 408, 873

Crocker, A. F., Jeong, H., Komugi, S., et al. 2009, MNRAS, 393, 1255

D’Onghia, E., Vogelsberger, M., \& Hernquist, L. 2013, ApJ, 766, 34

Evans, N. W., \& Collett, J. L. 1994, ApJ, 420, L67

García-Burillo, S., Sempere, M. J., Combes, F., Hunt, L. K., \& Neri, R. 2000, A\&A, 363, 869

Gorgas, J., Efstathiou, G., \& Salamanca, A. A. 1990, MNRAS, 245, 217

Johnston, E. J., Merrifield, M. R., Aragón-Salamanca, A., \& Cappellari, M. 2013, MNRAS, 428, 1296

Jore, K. P., Broeils, A. H., \& Haynes, M. P. 1996, AJ, 112, 438

Maraston, C., \& Strömbäck, G. 2011, MNRAS, 418, 2785

Morelli, L., Pompei, E., Pizzella, A., et al. 2008, MNRAS, 389, 341

Morelli, L., Corsini, E. M., Pizzella, A., et al. 2012, MNRAS, 423, 962

Pizzella, A., Corsini, E. M., Vega Beltrán, J. C., \& Bertola, F. 2004, A\&A, 424, 447

Pizzella, A., Morelli, L., Corsini, E. M., Dalla Bontà, E., \& Cesetti, M. 2013, A\&A, 560, A14

Pogge, R. W., \& Eskridge, P. B. 1987, AJ, 93, 291

Sandage, A., \& Bedke, J. 1994, The Carnegie Atlas of Galaxies (Washington: Carnegie Inst. Washington)

Sarzi, M., Falcón-Barroso, J., Davies, R. L., et al. 2006, MNRAS, 366, 1151

Thakar, A. R., Ryden, B. S., Jore, K. P., \& Broeils, A. H. 1997, ApJ, 479, 702

Thomas, D., Maraston, C., \& Bender, R. 2003, MNRAS, 339, 897

Vergani, D., Pizzella, A., Corsini, E. M., et al. 2007, A\&A, 463, 883

Worthey, G. 1994, ApJS, 95, 107 